\title{
MULHER EM EVIDÊNCIA: TRADUÇÃO DO CONHECIMEN- TO CIENTÍFICO PARA MULHERES POR MEIO DE UMA REDE SOCIAL
} Women in evidence: translation of scientific knowledge for women through a social network Mujeres en prueba: traducción de conocimientos cientificos para mujeres a través de una red social

\author{
Maria de Fátima Duarte Marinho 1 , Ana Beatriz da Fonseca Nunes², \\ Katia Maria Pereira ${ }^{3}$, Ayrlla Vytória Pereira ${ }^{4}$, Jayara Mikarla de Lira ${ }^{5}$, \\ Adriana Gomes Magalhães ${ }^{6}$, Grasiéla Nascimento Correia ${ }^{7}$
}

\section{RESUMO}

Durante a pandemia do novo coronavírus (SARS-CoV-2), as mulheres apresentaram dificuldade de acesso à saúde mediante o risco de transmissão do vírus. Devido a essa necessidade, foi criado o projeto de extensão "Mulher em Evidência - Conhecimento além dos muros do Trairi", que usou o Instagram para disponibilizar conteúdos relacionados à Saúde da Mulher, com embasamento científico e linguagem acessível. Foram publicados 72 conteúdos, alcançando 27.325 pessoas, tendo sido "O tabu da estética íntima" o tema mais curtido, com 236 curtidas. Inicialmente, os extensionistas relataram dificuldade para traduzir o conhecimento científico, entretanto, este projeto de extensão alcançou o objetivo de disseminar ciência de qualidade e promover saúde para mulheres.

Palavras-chave: Saúde da Mulher; Infecções por Coronavírus; Rede Social.

${ }^{1}$ Graduanda em Fisioterapia pela Universidade Federal do Rio Grande do Norte/Faculdade de Ciências da Saúde do Trairi. ${ }^{2}$ Graduanda em Fisioterapia pela Universidade Federal do Rio Grande do Norte/Faculdade de Ciências da Saúde do Trairi. ${ }^{3}$ Graduanda em Fisioterapia pela Universidade Federal do Rio Grande do Norte/Faculdade de Ciências da Saúde do Trairi.

\footnotetext{
${ }^{4}$ Graduanda em Enfermagem pela Universidade Federal do Rio Grande do Norte/Faculdade de Ciências da Saúde do Trairi. ${ }^{5}$ Graduanda em Enfermagem pela Universidade Federal do Rio Grande do Norte/Faculdade de Ciências da Saúde do Trairi. ${ }^{6}$ Doutora em fisioterapia, Professora adjunta da Universidade Federal do Rio Grande do Norte na Faculdade de Ciências da Saúde do Trairi.

${ }^{7}$ Doutora em fisioterapia, Professora adjunta da Universidade Federal do Rio Grande do Norte na Faculdade de Ciências da Saúde do Trairi.
} 


\section{ABSTRACT}

During the pandemic of the new coronavirus (SARS-CoV-2), women had difficulty accessing health care due to the risk of transmitting the virus. Due to this need, the extension project "Woman in Evidence - Knowledge beyond the walls of Trairi" was created, which used Instagram to provide content related to Women's Health, with scientific basis and accessible language. 72 contents were published, reaching 27,325 people and the most liked topic was "The taboo of intimate aesthetics" with 236 likes. Initially, extension workers reported difficulty in translating scientific knowledge, however this extension project achieved the objective of disseminating quality science and promoting health for women.

Keywords: Women's Health; Coronavírus Infections; Social Network.

\section{RESUMEN}

Durante la pandemia del nuevo coronavirus (SARS-CoV-2), las mujeres tuvieron dificultades para acceder a la atención médica debido al riesgo de transmitir el virus. Debido a esta necesidad, se creó el proyecto de extensión “Mujer en Evidencia - Conocimiento más allá de los muros de Trairi”, que utilizó Instagram para brindar contenido relacionado con la Salud de la Mujer, con base científica y lenguaje accesible. Se publicaron 72 contenidos, llegando a 27.325 personas y el tema más gustado fue "El tabú de la estética íntima" con 236 me gusta Inicialmente, los extensionistas reportaron dificultad para traducir el conocimiento científico, sin embargo este proyecto de extensión logró el objetivo de difundir ciencia de calidad y promover la salud de la mujer.

Palabras clave: Salud de la Mujer; Infecciones por Coronavírus; Red Social. 


\section{INTRODUÇÃO}

As mulheres representam a maioria da população brasileira $(51,80 \%)$ e são as principais usuárias do Sistema Único de Saúde (SUS) (IBGE, 2019), frequentando os serviços de saúde para atendimento próprio ou na figura de acompanhante de outros usuários (BRASIL, 2011). No entanto, o isolamento social ocasionado pela pandemia do novo coronavírus (SARS-CoV-2) causador da Corona Vírus Disease (COVID-19), levou a restrições no que diz respeito à procura por assistência à saúde devido aos riscos de contaminação e à alta transmissibilidade do vírus na comunidade, assim como em ambientes assistenciais (FERREIRA et al., 2020).

Não obstante, independentemente do momento de isolamento social vivido no Brasil, atualmente, continua vigente a Política Nacional de Atenção Integral a Saúde da Mulher (PNAISM), que preza por ações educativas, preventivas, de diagnóstico, tratamento e recuperação, englobando a mulher em sua integralidade. Assim, tal política tem como objetivos gerais a promoção de melhores condições de vida e saúde às mulheres brasileiras, a redução da morbimortalidade feminina, especialmente por causas evitáveis, e a qualificação de atenção à mulher no SUS (BRASIL, 2011).

Neste sentido, os espaços virtuais se tornaram uma alternativa ainda mais viável para ofertar atividades relacionadas à promoção à saúde devido ao crescimento do uso das mídias sociais, o que oportunizou novas formas de comunicação e de compartilhamento de conteúdo em diversas áreas da sociedade (MIRANDA; ROCHA, 2018). Dentre elas, destacam-se as ações de educação, uma vez que permitem o acesso ao conhecimento científico relacionado à mulher em seu aspecto biopsicossocial, espiritual e emocional, contribuindo com sua qualidade de vida sem que haja, na presente conjectura, o risco de contaminação.

No cenário atual, a educação em saúde por meio das redes sociais (MARTINS et al., 2020) tem desempenhado importantes papéis, como a divulgação de conhecimentos científicos de um modo acessível e compreensível ao público, aproximando o meio acadêmico da população e fazendo com que a universidade exerça um papel importante na disseminação da informação.

Outrossim, um ponto que se faz importante é o fato de que a escassez de fontes de conhecimento de qualidade, de fácil acesso e de entendimento pela comunidade pode predispor as mulheres a apresentarem doenças, complicações, e retardar por mais tempo a procura por atendimento de saúde. Neste sentido, atualmente tem se adotado a Tradução do Conhecimento (TC), que é definida como a síntese, o intercâmbio e a aplicação do conhecimento pelas partes interessadas para acelerar os benefícios da inovação global e local dos sistemas de saúde e a melhoria da saúde das pessoas (FERRAZ; PEREIRA; PEREIRA, 2019). 
Assim, as redes sociais, por serem fontes de informação de acesso fácil, econômicas e eficazes, vêm sendo utilizadas de forma ampla em todo o mundo como estratégia para TC (VERMELHO et al., 2014), uma vez que milhares de pessoas buscam diariamente por informações de todos os tipos, sejam elas relativas a esportes, doenças ou até a desastres naturais (GONZÁLEZ-PADILLA; TORTOLERO-BLANCO, 2020). Além disso, as mídias sociais também servem como meio de aproximação entre as pessoas, reduzindo o isolamento e o tédio, fatores esses associados a transtornos de ansiedade e sofrimento, no que se percebe a relevância da recomendação do seu uso durante o isolamento social, tanto para redução de estresse quanto para melhoria do impacto psicológico (GONZÁLEZ-PADILLA; TORTOLERO-BLANCO, 2020).

Nesse sentido, o projeto de extensão "Mulher em Evidência - Conhecimento além dos muros do Trairí" foi criado com o intuito de proporcionar informações relacionadas à Saúde da Mulher nos diferentes ciclos da vida, atingindo mulheres de diferentes níveis sociais e educacionais e contribuindo com o desenvolvimento da comunidade por meio da adoção de uma linguagem consistente, acessível e compreensível a todos como instrumento de divulgação do conhecimento por meio eletrônico.

\section{OBJETIVOS}

Este trabalho tem como objetivo relatar as ações do projeto "Mulher em Evidência - Conhecimento além dos muros do Trairí", que proporcionou informações relacionadas à Saúde da Mulher nos diferentes ciclos da vida, por meio de uma linguagem acessível utilizando a rede social Instagram.

\section{METODOLOGIA}

Trata-se, portanto, de um estudo descritivo do tipo relato de experiência de abordagem quali-quanti vinculado ao projeto de extensão "Mulher em Evidência - Conhecimento além dos muros do Trairí", realizado pela Faculdade de Ciências da Saúde do Trairí (FACISA), campus da Universidade Federal do Rio Grande do Norte (UFRN), durante o período de março a dezembro de 2020. Os extensionistas eram discentes do curso de fisioterapia e enfermagem, que estavam entre o $4^{\circ} \mathrm{e}$ $9^{\circ}$ semestre do curso e desempenharam as atividades na rede social Instagram com a finalidade de contribuir com a promoção de saúde relacionada à Saúde da Mulher nos diferentes ciclos da vida.

A primeira atividade do projeto de extensão foi o desenvolvimento de um perfil no Instagram intitulado "Mulher em Evidência - @mulheremevidenciaufrnfacisa" em março de 2020. Inicial- 
mente, foi realizada a criação do perfil e da identidade visual da página (logotipo e os layouts dos posts e stories). A partir desses direcionamentos, as postagens abordavam temas referentes à anatomia do sistema reprodutor feminino, obstetrícia, ginecologia, urologia, oncologia, disfunções sexuais, educação sexual, dentre outros temas livres que pudessem surgir, como assuntos relacionados ao SARS-COV-2.

Os temas abordados eram definidos com um mês de antecedência, de acordo com o interesse dos extensionistas e das sugestões do público por meio de caixa de perguntas nos stories do Instagram. Os conteúdos abordados eram provenientes de artigos científicos, redigidos pelos extensionistas e corrigidos pelos docentes que participavam do projeto para que fossem publicados com uma linguagem simples, clara, objetiva e coesa para atingir adequadamente o público-alvo. Estes conteúdos eram publicados duas vezes na semana no feed da rede social e, durante a semana, havia interação com o público que seguia a página, os seguidores, por meio da ferramenta stories, em que eram postadas informações e realizados jogos de perguntas e respostas sobre os temas das postagens.

\section{RESULTADOS E DISCUSSÃO}

\section{Perfil do público-alvo}

Durante esse período, no perfil do Instagram, foram publicados 72 conteúdos, o que atingiu 27.325 pessoas e perfez 720 seguidores, sendo o público, em sua maioria mulheres $(92,10 \%)$, proveniente de Natal/RN (16,60\%) e Santa Cruz/RN (16,00\%). Além disso, 43,10\% destas mulheres possuíam faixa etária entre 18 e 24 anos.

De acordo com Karatsoli e Nathanail (2020), o público feminino é mais receptivo às informações fornecidas pelas redes sociais. Twenge e Martin (2020) tentaram investigar as diferenças de gênero no uso da mídia social avaliando adolescentes de 13 a 18 anos nos Estados Unidos e no Reino Unido. Assim, os resultados mostraram que as meninas adolescentes gastam mais tempo em smartphones, mídias sociais, mensagens de texto e uso geral do computador em comparação aos meninos. No entanto, apesar de muitos jovens utilizarem as redes sociais como fontes facilmente acessíveis, a confiabilidade das informações fornecidas é questionável (AILLERIE; MCNICOL, 2018; FLANAGIN; METZGER, 2010).

As redes sociais apresentam algumas características muito importantes, como: amplo e fácil 
acesso, baixo custo, multimídia, interatividade, utilização sem a necessidade de habilidades e conhecimentos, comunicação instantânea e continuidade (HAMPTON; WELLMAN, 2003). Desse modo, estas redes sociais, como por exemplo o Instagram, fornecem oportunidades para diferentes formas de troca comunicativa (HENDRIKS et al., 2020). Além disso, podem ser conceituadas por meio do construtivismo com características variáveis de aprendizado formal e informal (GREENHOW; LEWIN, 2016), deixando de serem vistas apenas como uma ferramenta de entretenimento e ganhando notoriedade por criarem ainda mais oportunidades para os indivíduos se conectarem com a ciência (BROSSARD; SCHEUFELE, 2013).

Assim, lidar com a complexidade do conhecimento científico em um ambiente de mídias digitais é um desafio (KIENHUES; JUCKS; BROMME, 2020), especialmente porque esse conhecimento é frequentemente comunicado de maneira muito formal, principalmente quanto ao uso da linguagem. Logo, as plataformas digitais oferecem aos leigos a oportunidade de aprender sobre a ciência em vários formatos e em uma linguagem muito mais acessível e envolvente (HENDRIKS et al., 2020).

\section{Principais publicações}

A figura 1 representa as principais publicações que foram postadas a cada mês, sendo que destas as mais curtidas abordavam temas como: “O tabu da estética íntima” (236 curtidas), "Quais são as infecções vaginais que mais acometem as mulheres?" (178 curtidas) e "Coletor menstrual" (177 curtidas). Dessa forma, infere-se que estes temas podem despertar mais interesse no público feminino por abordarem assuntos relevantes e pouco discutidos, mas que estão presentes na vida das mulheres. 
Figura 1 - Principais publicações realizadas entre os meses de abril a dezembro de 2020

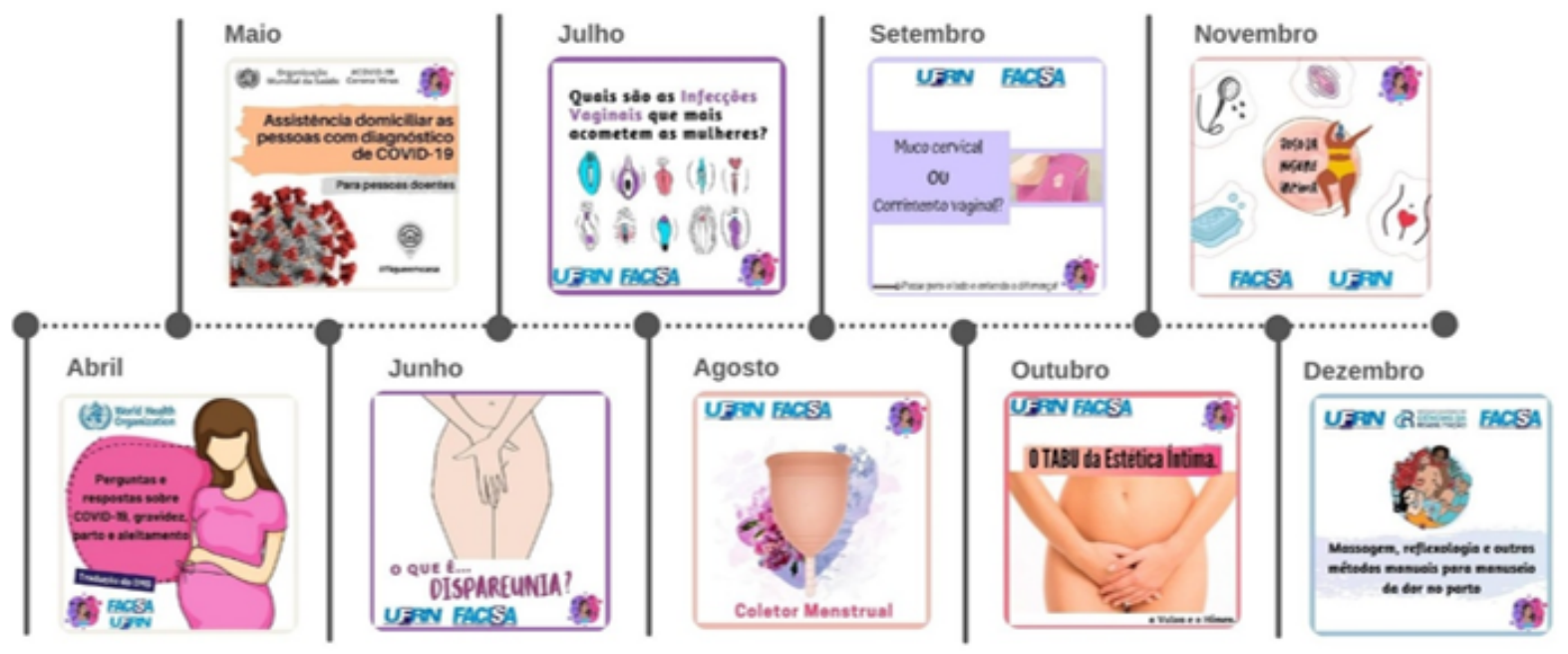

Fonte: Autoria própria (2021).

Dentre os assuntos mais curtidos, o tema "O tabu da estética íntima” obteve destaque, temática essa que levanta questões pertinentes ao embelezamento da região íntima/aparência da genitália.

Uma vez que a vulva é um símbolo de feminilidade (VASCONCELOS, 2013), cada vez mais o corpo passa a sujeitar-se à exploração econômica da erotização e dos produtos embelezadores (SAMPAIO, FERREIRA, 2009). Desta forma, a postagem discorreu sobre a importância de saber que existem vários tipos de vulvas e sobre o empoderamento da mulher sobre essa questão.

As infecções do trato reprodutivo (ITR), incluindo infecções sexualmente transmissíveis (IST), são temas que merecem cuidado especial por parte da saúde pública e que foram abordados na postagem “Quais são as infecções vaginais que mais acometem as mulheres?”. Estima-se, pois, que uma grande proporção dos casos curáveis de IST ocorrem em mulheres em idade reprodutiva (WORLD HEALTH ORGANIZATION, 2005), bem como as piores consequências dessas infecções recaem principalmente sobre elas (WORLD HEALTH ORGANIZATION, 2006). Desta forma, é de suma importância que as mulheres tenham consciência das infecções vaginais que mais as acometem.

Ademais, outro tema que obteve destaque diz respeito ao uso do "Coletor menstrual", que surge como um produto alternativo aos mais tradicionais métodos menstruais e é inserido na vagina, com o sangue coletado em recipiente, que pode ser de silicone, borracha, látex ou elastômero (VAN EIJK et al., 2019). Neste sentido, foi feita uma enquete no Instagram com a pergunta "Você já usou coletor menstrual?", por meio da qual 78\% das mulheres responderam que "Não". Além disso, foi 
realizada uma caixa de perguntas com o seguinte questionamento às seguidoras: "Você tem dúvida sobre coletor menstrual?", e, em seguida, foram esclarecidas dúvidas sobre como escolher o tamanho adequado, como inserir/retirar e como higienizar adequadamente o recipiente, o que demonstra que esse tema é ainda desconhecido pela maioria das mulheres e que esse público carece de informação de qualidade.

\section{Interações dos seguidores}

Em relação às interações dos seguidores, as postagens obtiveram um alcance de 27.325 pessoas/contas, 4.470 curtidas, 287 comentários, 760 compartilhamentos e 504 salvamentos, como mostra a figura 2. Quanto às interações nos stories, foram realizados alguns jogos de perguntas e respostas referentes aos temas das postagens, sendo os principais: anatomia do sistema reprodutor feminino (177 respostas), métodos contraceptivos (145 respostas), sororidade e feminismo (112 respostas) e incontinência urinária (64 respostas). As postagens da página também foram utilizadas no atendimento remoto das pacientes do estágio supervisionado de fisioterapia em atenção à saúde da mulher da UFRN/FACISA.

Figura 2 - Temas de postagens e dados de alcance e curtidas

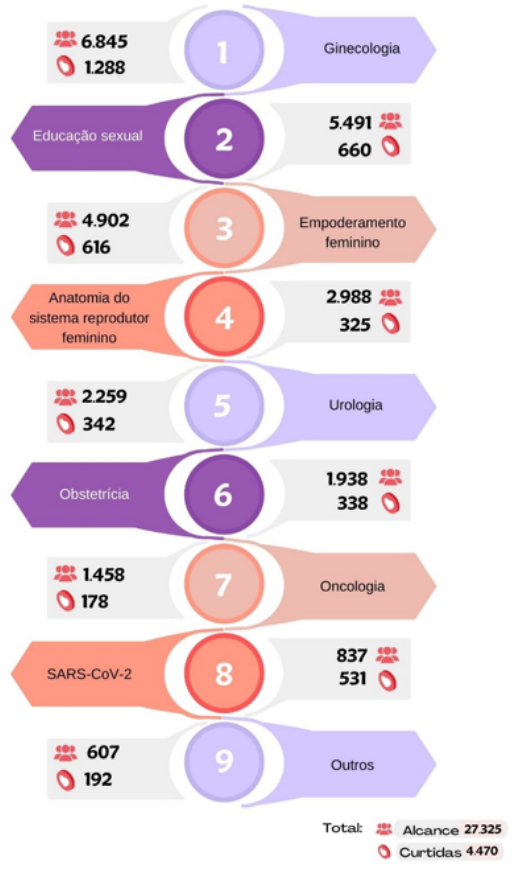

Fonte: Autoria própria (2021). 


\section{Publicações sobre a COVID-19}

Durante os meses de abril e setembro de 2020, no início da pandemia por SARS-COV-2, o perfil realizou diversas publicações relacionadas a essa temática, algumas delas como: "Perguntas e respostas sobre COVID-19, gravidez, parto e aleitamento", "Cuidados da mamãe e o bebê durante a pandemia", "Violência contra a mulher durante a pandemia", "Assistência domiciliar às pessoas com diagnóstico de COVID-19", "Saúde do idoso em tempos de pandemia", entre outras. Essas postagens foram estimuladas pela universidade e representou uma forma de disseminar conhecimento de qualidade, com evidência científica para a sociedade, e de tentar combater as fakenews.

Assim sendo, a mídia social tem sido usada em várias aplicações de saúde (SAMPSON et al., 2013), como, por exemplo, durante a pandemia do SARS-COV-2: à medida que o vírus se espalhava pelo mundo, também se espalhavam as informações e discussões sobre ele nas redes sociais (LU; ZHANG, 2020). Durante as crises de saúde pública, essas informações também podem ajudar os pesquisadores e profissionais de saúde a avaliar o medo do público, a combater a desinformação, a aumentar a conscientização e a fornecer instruções e treinamento direcionados (SELTZER et al., 2015).

\section{Dificuldades encontradas e feedbacks}

Durante a realização do projeto, foram encontradas algumas dificuldades pelos extensionistas. Com relação à criação do conteúdo, foi preciso selecionar os assuntos que interessassem as mulheres e produzir postagens que fossem visualmente criativas e precisas para se destacarem em meio a outras postagens da rede social. Também foi necessário readequar a linguagem encontrada nos artigos científicos para uma linguagem simples e objetiva, o que demandava mais habilidades e tempo dos discentes. Esse processo de tradução do conhecimento contribuiu com a capacidade dos alunos de se comunicarem com seus pacientes e de repassarem informações de maneira mais clara, o que auxilia na divulgação do conhecimento científico para a comunidade.

Ao final do ano, o público da página respondeu a uma caixa com a pergunta "Você conseguiu aprender algo com nossas postagens?", momento em que recebemos como feedback respostas como "Foi ótimo, adquiri muito conhecimento", “Aprendi demais", "Compartilhar os posts de vocês virou rotina", "Bastante, amei os posts, empoderadores demais", "Consegui perceber que eu já tive bexiga hiperativa”, entre outros. Não recebemos nenhuma crítica construtiva ou comentários negativos. 


\section{CONSIDERAÇÕES FINAIS}

Nota-se, portanto, que este projeto de extensão contribuiu com a disseminação de informação científica pela universidade para a comunidade, de forma fácil, clara e coesa, por meio de uma rede social. Ao proporcionarmos informações de qualidade para esse público, aproximamos este da universidade e beneficiamos essas mulheres, uma vez que o acesso a informações sobre o autocuidado voltado à saúde da mulher auxilia na prevenção e promoção de sua saúde. Além disso, os extensionistas também foram beneficiados, uma vez que aprenderam a traduzir o conhecimento científico para a sociedade.

Assim, espera-se que esse projeto continue divulgando a ciência voltada para o público feminino, alcançando um número maior de pessoas.

\section{REFERÊNCIAS}

AILLERIE, Karine; MCNICOL, Sarah. Are social networking sites information sources? Informational purposes of high-school students in using SNSs. Journal of Librarianship and Information Science, v.50, n.1, p.103-114, 2018. https://doi.org/10.1177/0961000616631612.

BRASIL. Ministério da Saúde. Secretaria de Atenção à Saúde. Departamento de Ações Programáticas Estratégicas. Política Nacional de Atenção Integral à Saúde da Mulher: Princípios e Diretrizes - 1. ed., 2. reimpr. Brasília: Editora do Ministério da Saúde, 2011. Disponível em: https://bvsms. saude.gov.br/bvs/publicacoes/politica_nacional_mulher_principios_diretrizes.pdf. Acesso em: 10 maio. 2021.

BROSSARD, Dominique; SCHEUFELE, Dietram. Science, new media, and the public. Science, v.339, n.6115, p. 40-41, 2013. https://doi.org/10.1126/science.1232329.

FERRAZ, Lucimare; PEREIRA, Rui Pedro Gomes; PEREIRA, Altamiro Manuel Rodrigues da Costa. Tradução do Conhecimento e os desafios contemporâneos na área da saúde: uma revisão de escopo. Saúde em Debate, v. 43, n. 2, p. 200-216, 2019. http://dx.doi.org/10.1590/ 0103-11042019s215. 
FERREIRA, Verônica Clemente; SILVA, Mariana Regazzi Ferreira; MONTOVANI, Elisa Hypólito; COLARES, Larissa Gobbi; RIBEIRO, Aridiane Alves; STOFEL, Natália Sevilha. Saúde da Mulher, Gênero, Políticas Públicas e Educação Médica: agravos no contexto de pandemia. Revista Brasileira de Educação Médica, v. 44, n. 1, p. 1-8, 2020. http://dx.doi.org/10.1590/1981-5271v44. supl.1-20200402.

FLANAGIN, Andrew J.; METZGER, Miriam J.. Kids and Credibility: An Empirical Examination of Youth, Digital Media Use, and Information Credibility. Cambridge, MA and London: The MIT press, 2010. https://doi.org/10.1080/1369118x.2011.627182.

GONZÁLEZ-PADILLA, Daniel A.; TORTOLERO-BLANCO, Leonardo. Social media influence in the COVID-19 Pandemic. International Braz J Urol, v. 46, n. 1, p. 120-124, 2020. https://doi. org/10.1590/s1677-5538.ibju.2020.s121.

GREENHOW, Christine; LEWIN, Cathy. Social media and education: reconceptualizing the boundaries of formal and informal learning. Learning, Media and Technology, v. 41, n. 1, p. 6-30, 2016. https://doi.org/10.1080/17439884.2015.1064954.

HAMPTON, Keith; WELLMAN, Barry. Neighboring in Netville : How the Internet Supports. City \& Community, v. 2, n. 4, p. 277-311, 2003. https://doi.org/10.1046/j.1535-6841.2003.00057.x.

HENDRIKS, Friederike; MAYWEG-PAUS, Elisabeth; FELTON, Mark; IORDANOU, Kalypso; JUCKS, Regina; ZIMMERMANN, Maria. Constraints and Affordances of Online Engagement With Scientific Information-A Literature Review. Frontiers in Psychology, v. 11, p. 1-21, 2020. https://doi.org/10.3389/fpsyg.2020.572744.

IBGE - Instituto Brasileiro de Geografia e Estatística Diretoria de pesquisa. Características gerais dos domicílios e dos moradores 2019. Pesquisa Nacional por Amostra de Domicílios Contínua - PNAD Contínua. Disponível em: https://educa.ibge.gov.br/jovens/conheca-o-brasil/populacao/ 18320-quantidade-de-homens-e-mulheres.html. Acesso em: 11 maio. 2021. 
KARATSOLI, Maria; NATHANAIL, Eftihia. Examining gender differences of social media use for activity planning and travel choices. European Transport Research Review, v. 12, n.44, 2020. https://doi.org/10.1186/s12544-020-00436-4.

KIENHUES, D.; JUCKS, R.; BROMME, R. Sealing the gateways for post-truthism: Reestablishing the epistemic authority of science. Educational Psychologist, v. 55, n. 3, p. 144-154, 2020. https://doi.org/10.1080/00461520.2020.1784012.

LU, Yue; ZHANG, Leiliang. Social media WeChat infers the development trend of COVID-19. Journal of Infection, v. 81, n. 1, p. e82-e83, 2020. https://doi.org/10.1016/j.jinf.2020.03.050.

MARTINS, Vivian; ALMEIDA, Joelma. Educação em tempos de pandemia no brasil: saberes fazeres escolares em exposição nas redes e a educação on-line como perspectiva. Revista docência e cibercultura, v. 4, n.2, p.215-224, 2020. https://doi.org/10.12957/redoc.2020.51026.

MIRANDA, Fernanda Santana; ROCHA, Dais Gonçalves. O uso do Facebook na promoção da saúde: uma revisão bibliográfica sobre empoderamento e participação popular. Revista Eletrônica de Comunicação, Informação e Inovação em Saúde, v. 12, n. 2, p. 231-243, 2018. http://dx.doi. org/10.29397/reciis.v12i2.1331.

SAMPSON, Margaret; CUMBER, Jordi; LI, Claudia; POUND, Catherine M.; FULLER, Ann; HARRISON, Denise. A systematic review of methods for studying consumer health YouTube videos, with implications for systematic reviews. PeerJ, v.1, n.1, p. 1-16, 2013. https://doi. org/10.7717/peerj.147.

SAMPAIO, R. P. A.; FERREIRA, R. F. Beleza, identidade e mercado. Psicologia em Revista, Belo Horizonte, v. 15, n. 1, p. 120-140, abr. 2009.

SELTZER, EK; JEAN, NS; KRAMER-GOLINKOFF, E; ASCH, DA; MERCHANT, RM. The content of social media's shared images about Ebola: A retrospective study. Public Health, v. 129, n. 9, p. 1273-1277, 2015. https://doi.org/10.1016/j.puhe.2015.07.025. 
TWENGE, Jean M.; MARTIN, Gabrielle N. Gender differences in associations between digital media use and psychological well-being: Evidence from three large datasets. Journal of Adolescence, v. 79, p. 91-102, 2020. https://doi.org/10.1016/j.adolescence.2019.12.018

VAN EIJK, Anna Maria et al. Menstrual cup use, leakage, acceptability, safety, and availability: a systematic review and meta-analysis. The Lancet Public Health, v. 4, n. 8, p. e376-e393, 2019. https://doi.org/10.1016/S2468-2667(19)30111-2.

VASCONCELOS, Carmen. Cirurgia íntima ajuda a vencer medos e melhora a atividade sexual. IBahia, 2013. Disponível em: https://www.ibahia.com/detalhe/noticia/cirurgia-intima-ajuda-a-vencer-medos-e-melhora-a-atividade-sexual/. Acesso em: 12 abr. 2021.

VERMELHO, Sônia Cristina; VELHO, Ana Paula Machado; BONKOVOSKI, Amanda; PIROLA, Alisson. Refletindo sobre as redes sociais digitais. Educ. Soc., v. 35, n. 126, p. 179-196, 2014. http://dx.doi.org/10.1590/S0101-73302014000100011

WORLD HEALTH ORGANIZATION. Prevention and management of sexually transmitted and reproductive tract infections: integrated management of pregnancy and childbirth (IMPAC). Standards for Maternal and Neonatal Care, 2006. Disponível em: https://www.who.int/reproductivehealth/publications/maternal_perinatal_health/prevention_mngt_stis.pdf?ua=1. Acesso em: 10 maio. 2021.

WORLD HEALTH ORGANIZATION. Sexually transmitted and other reproductive tract infections: a guide to essential practice. World Health Organization, 2005. Disponível em: https:// www.who.int/reproductivehealth/publications/rtis/9241592656/en/. Acesso em: 10 maio. 2021. 